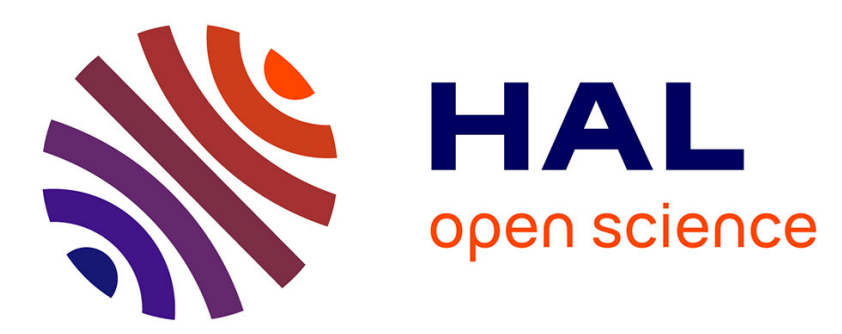

\title{
Analysis of Grains Distribution Effects on the Magnetic Spectra of Polycrystalline Ferrites
}

\author{
J. Jankovskis, V. Yurshevich, G. Rankis
}

\section{To cite this version:}

J. Jankovskis, V. Yurshevich, G. Rankis. Analysis of Grains Distribution Effects on the Magnetic Spectra of Polycrystalline Ferrites. Journal de Physique IV Proceedings, 1997, 07 (C1), pp.C1-203C1-204. 10.1051/jp4:1997176 . jpa-00255123

\section{HAL Id: jpa-00255123 https://hal.science/jpa-00255123}

Submitted on 1 Jan 1997

HAL is a multi-disciplinary open access archive for the deposit and dissemination of scientific research documents, whether they are published or not. The documents may come from teaching and research institutions in France or abroad, or from public or private research centers.
L'archive ouverte pluridisciplinaire HAL, est destinée au dépôt et à la diffusion de documents scientifiques de niveau recherche, publiés ou non, émanant des établissements d'enseignement et de recherche français ou étrangers, des laboratoires publics ou privés. 


\title{
Analysis of Grains Distribution Effects on the Magnetic Spectra of Polycrystalline Ferrites
}

\author{
J. Jankovskis, V. Yurshevich and G. Rankis
}

Riga Technical University, 12 Azenes str., LV-1048 Riga, Latvia

\begin{abstract}
The effects of grain size distribution of soft polycrystalline ferrites on its domain wall initial susceptibility magnetic spectra are analyzed. It is shown that absorption component of spectrum may be inhomogeneously broadened, and that it formally reproduces the grain size distribution density function. Explicit relations for the absorption component and some characteristic parameters of spectrum are presented.
\end{abstract}

\section{INTRODUCTION}

The initial magnetic susceptibility (IMS) $\chi_{i}$ of soft polycrystalline ferrites (PF) decisively depends on their granular (micro-) structure - on average grain size $D_{a}$ and size distribution. Dependencies of the first type have been much investigated (most systematically by A. Globus a.o. from Laboratory de Magnetisme, C.N.R.S.). Prevailing conclusions are that $\chi_{i}$ is linear with $D_{a}$; nonlinearity usually appears when samples with the wider range of $D_{a}$ sizes are analyzed. The second type of dependencies - influences of grain size distribution - are less investigated. We try to extend the already known as well as our previous [1] studies for linear region of $\chi_{i}$ in radio-frequency range where domain wall (DW) processes are dominating. In our analysis we assume that magnetism in elementary volumes of PF material is in relation with grains (much the same as in A. Globus' models). We also are forced to make a guess a priori about possible relations used as interior ones (mostly on the base of the data gained from many PF samples). Validity of these assumptions is ascertained later on from derived solutions.

\section{THEORETICAL ANALYSIS}

We assume that in PF there are elementary volumes $v(\chi)$ with susceptibility $\chi$ (continuously distributed in the range $\left.\chi_{\min . . .} \chi_{\max }\right)$. After relating $\chi$ with the definite value of $\mathrm{D}$, it is possible to write for the quasi-static IMS $\chi_{i}(0)$ of isotropic

sample: $\quad \chi_{i}(0)=\frac{2}{3} \int_{\chi \min }^{\chi \max } \chi \frac{v(\chi)}{v_{a}} \varphi(\chi) d \chi=\frac{2}{3} \int_{D \text { min }}^{D \max } \chi(D) \frac{v(D)}{v_{a}} \varphi(D) d D$

where $v_{o}$ is the average of elementary volumes, $\varphi(\chi)$ and $\varphi(D)$ are density functions. For explicit evaluation of $\chi_{i}(0)$ we also assume that $\quad \chi(D)=g D ; \quad v(D)=a D^{N}$

where $g, a, N$ are const., and $\varphi(D)$ is log-normal [2] with median $D_{\text {med }}$ and standard deviation $\sigma$ of $\log D$. Insertion of Eq. (2), $\varphi(D)$ and $v_{c r}=a D_{m e d}^{N} \exp \left(0.5 N^{2} \sigma^{2} \mathrm{In}^{2} 10\right)$ in Eq.(1) and the assumption that $D_{\min } \rightarrow 0, D_{\max } \rightarrow \infty$ (that sets up negligible error [3]) allow to represent IMS in typical linear form with constant's $G$ value elucidating the influence of grains dispersion on $\chi_{i}(0)$ :

$$
\chi_{i}(0)=(2 / 3) g \exp \left(N \sigma^{2} \ln ^{2} 10\right)=G D_{a} \text {. }
$$

To investigate dependence of IMS on frequency $f$, we assume that volumes $v(\chi)$ contain domain wall (walls), displacements of which are responsible for $\chi$ value and which are resonating on frequency $f_{0}$. This allows to replace Eq.(1) by

$$
\chi_{i}^{\bullet}(f)=\frac{2}{3} \int_{0}^{\infty} \chi^{*}\left(f, f_{0}\right)\left[v\left(f_{0}\right) / v_{a}\right] \varphi\left(f_{0}\right) d f_{0} .
$$

Eq.(4) implicitly represents both parts of complex IMS $\chi_{i}^{*}(f)$ - dispersion (DP) $\chi_{i}^{*}(f)$ and absorption (AP) $\chi_{i}(f)$ ones of magnetic spectrum (MS). To estimate these parts, we use harmonic oscillator approximation for DW displacement processes [4]; this allows to represent imaginary part of $\chi^{\circ}\left(f, f_{0}\right)$ in the form:

$$
\chi^{\prime \prime}\left(f, f_{0}\right)=2 \chi_{0} \xi f f_{0}^{3} /\left[\left(f_{0}^{2}-f^{2}\right)^{2}+4 \xi^{2} f^{2} f_{0}^{2}\right]
$$


where $\chi_{0}$ is the static IMS of elementary oscillator; $\xi$-dimensionless damping coefficient. Value of $\chi_{0}$ is related to $f_{0}:$ in agreement with many MS of PF samples for which more or less is fulfilled relation [4]: $\chi_{0}(0) f_{u} / M_{s} \approx 4 \pi=C$ where $f_{u}$ maximum absorption frequency, $M_{S}$ - magnetization, $C$-constant, we may assume that

$$
\chi_{0}=C_{0} M_{S} / f_{0} ; \quad v\left(f_{0}\right)=a\left(C_{0} M_{S} / g f_{0}\right)^{N}
$$

(The latter relation comes from Eq.(2) when there is assumed that in PF $f_{0} \sim 1 / D$ and for PF sample $f_{u} \sim 1 / D_{a}$ ). Density function $\varphi\left(f_{0}\right)$ is still $\log$-normal with the same $\sigma$ and $f_{\text {med }}=C_{0} M s / g D_{\text {med }}$. Insertion of Eqs. (5), (6) and $\varphi\left(f_{0}\right)$ in Eq.(4) allows to represent AP $\chi \chi^{\prime \prime}(f)$ in integral form. From this form it is possible to derive a more useful approximate relation, if in the elementary absorption line's Eq.(5) even part and its integral is replaced by $\delta$-function. This replacement allows to obtain: $\quad \chi_{i}(f)=A f^{-(N+1)} \exp \left[-\left(\log f-\log f_{0 \text { ned }}\right)^{2} / 2 \sigma^{2}\right]$

where $A$ is const. Differentiation of Eq.(7) allows to obtain: $\quad f_{u}=f_{0 m e d} \exp \left[-\sigma^{2}(N+1) \ln ^{2} 10\right.$.

$$
\text { From this relation and Eq.(3), it follows that } \quad x_{i}(0) f_{v} / M_{S}=(2 / 3) C_{0} \exp \left(-0.5 \sigma^{2} \ln ^{2} 10\right) \text {. }
$$

AP $\chi \hat{i}(f)$ may be presented in a form that is more convenient for applications, using Eqs.(7), (8) and the aforementioned definitions of constants:

$$
\chi_{i}(f)=\chi_{i \max }^{\prime \prime} \exp \left[-\left(\log f_{u} / f\right)^{2} / 2 \sigma^{2}\right]
$$

where $\chi \ddot{i n a x}$ stands for maximum absorption at $f_{11}$ and has value:

$$
\chi_{i \max }^{*}=\chi_{i}(0) / 3.67 \sigma \text {. }
$$

The value of $\sigma$, when Eq.(10) is fitted for real MS, may be evaluated from characteristics $f_{1 / L}$ and $f_{u}$ (viewed as experimental ones): $\quad \sigma=(1 / \sqrt{2 \ln L}) \log \left(f_{u} / f_{1 / L}\right)$, where $f_{1 / L}<f_{\nu}$ and corresponds to definite absorption level $L=\chi_{i m a x} / \chi_{i}^{*}\left(f_{1 / L}\right)$. Explicit expression for DP $\chi_{i}^{\prime}(f)$ from Eq.(4) is not get yet, but it is possible to calculate DP from AP, using Kramers-Kronig relations (KKR). For presentation of DP (and also for the experimental MS quality estimation), we use our computer program for KKR.

\section{APPLICATIONS}

Normalized MS, with AP calculated by Eq.(10) and DP from AP by KKR for $f_{u}=1, \chi_{i \text { max }}=1$ and several values of $\sigma$, is shown in Fig. la. The curves show the typical features which can be observed in real MS, e.g., resonance - for the lower value $\sigma$ and relaxation - for the greater one (for simple relaxation MS [4] from Eq.(11) $\sigma \approx 055$ ). When using the relations for
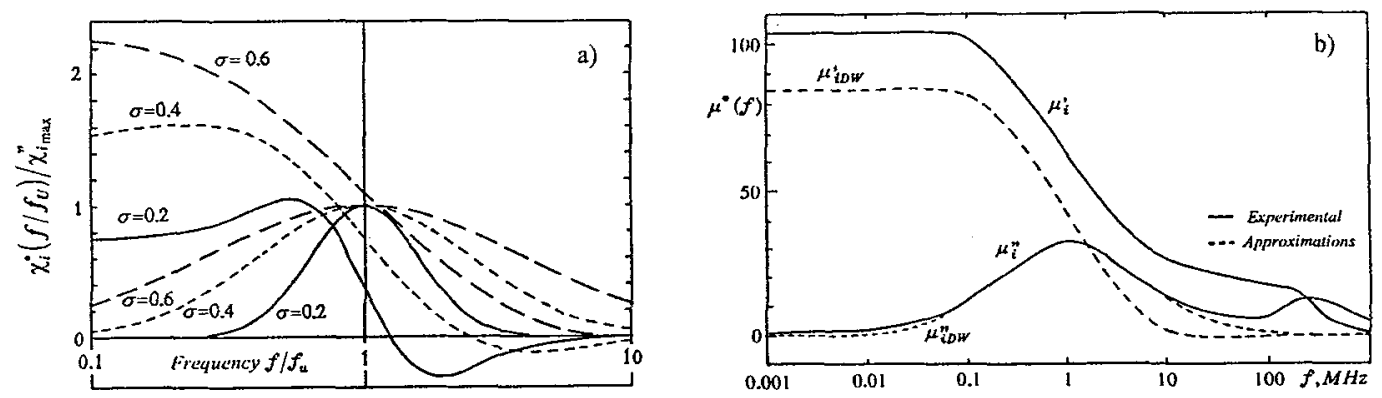

Fig. 1. Magnetic spectra of PF: a) theoretical, b) approximation of YIG spectrum from [5].

approximation of real MS, it must be noted that in the above analysis we have dealt only with typical DW resonance $/$ relaxation region. Such regions are well-presented only in a few spectra, e. g., in similar ones for YIG in [5] and [6] (besides they adequately obey KKR; this is not so with many other MS). Approximation for the first spectrum is in reasonably good agreement with the data (Fig. $1 \mathrm{~b}, \sigma=0.7$, doted lines); for the second one agreement is not so good for frequencies $f>f_{\prime \prime}$, because experimental AP is non-symmetrical in log-scale. For both approximations DW component $\chi_{i D W}(0)$ fulfills Eq.(11). Conclusions. The analysis show that AP of PF magnetic spectrum may be considerably broadened by grain size distribution (inhomogeneous line broadening), and that it formally reproduces the grain size density function.

\section{References}

[1] J. Jankovskis, Radioelectronics \& Telecommunications, No.19 (1992), 64-72 (Riga, in Russian).

[2] S. K. Kurtz, F.M.A. Carpay, J. Appl. Phys., 51 (1980), No.11, 5725-5754.

[3] C. Borghese, J. Appl. Phys., 44 (1973), No.8, 3746-3751.

[4] M. Rosenberg, C. Tânâsoiu, In: Magnetic Oxides. Ed. D.J. Craik. J. Wiley \& Sons, 1975, Part 2, 483-573.

[5] D. J. Epstein, B. Franckiewicz, In: Standley K. J. Oxide Magnetic Materials. Oxford, Clarendon Press, 1962.

[6] M. Guyot, T. Merceron, V. Cagan, A. Messekher, Phys. Stat. sol.(a), 106 (1988), No.2, 595-612. 